\title{
Transcatheter left atrial appendage occlusion: patient selection and perspectives
}

This article was published in the following Dove Press journal:

Research Reports in Clinical Cardiology

5 July 2016

Number of times this article has been viewed

\section{Wojciech CWąsek}

Cardiology Department, Military Institute of Medicine, The Central Teaching Hospital of the Ministry of Defense, Szaserów, Warsaw, Poland
Correspondence:Wojciech CWąsek Cardiology Department, Military Institute of Medicine, The Central Teaching Hospital of the Ministry of Defense, Szaserów 128, 0I-14I Warsaw, Poland Email wwasek@wim.mil.pl

\begin{abstract}
The issues of stroke prevention in patients with nonvalvular atrial fibrillation are extremely important. Despite the availability of warfarin and novel non-vitamin K oral anticoagulants, many problems remain. The necessity of long-term therapy with the incremental lifetime risk of bleeding, underuse of anticoagulation treatment in population at high embolic risk due to contraindications or drug interactions, along with the lack of treatment efficacy, represent the vast spectrum of unachieved clinical needs. The local, device-based, preventive treatment targeting left atrial appendage occlusion was designed to fulfill the unmet clinical expectations and possibly constitutes an alternative to anticoagulation therapy. The short- and long-term results of the WATCHMAN Left Atrial Appendage Closure Device for Embolic PROTECTion in Patients with Atrial Fibrillation (PROTECT AF) randomized trial were promising; however, data derived from the second randomized Prospective Randomized Evaluation of the WATCHMAN LAA Closure Device in Patients With Atrial Fibrillation Versus Long Term Warfarin Therapy (PREVAIL) trial were diverging, leaving uncertainty in appendage closure efficacy evaluation in comparison to anticoagulation. The totality of data, however, allowed the US Food and Drug Administration to register the WATCHMAN device for embolic prevention in patients deemed by their physicians to be suitable for warfarin and have appropriate rationale to seek a nonpharmacologic alternative to anticoagulation. In contrary to the Food and Drug Administration limited device registration, European implementation of this technology based on CE marking and the European Heart Rhythm Association and the European Association of Percutaneous Cardiovascular Interventions expert consensus allows for much wider clinical application. The aim of this paper is to present the pros and cons of this novel therapy, shed light on the complexity of data derived from clinical trials leading to final decisions on device registrations, and deliver evidencebased and expert opinion-based principles for proper selection of patients benefiting from left atrial appendage occlusion.
\end{abstract}

Keywords: atrial fibrillation, appendage occlusion, patient selection

\section{The need for an alternative to anticoagulant therapy}

The threefold rise in prognosis of nonvalvular atrial fibrillation (NVAF) morbidity till the year 2050, with proved independent twofold to threefold higher risk of death in long-term follow-up forces the social importance of the disease. ${ }^{1}$ According to Framingham Study findings, patients with the history of stroke attributable to NVAF have $63 \%$ annual risk of dying. The current standard of stroke and death prevention strategy is based on lifelong oral anticoagulation mainly using vitamin K antagonists 
(VKA), having, at a maximum, over $60 \%$ relative risk reduction compared with placebo, and more recently on novel anticoagulants (NOACs). ${ }^{2}$ Antiplatelet therapy to prevent vascular events in NVAF patients presents disappointing results. The combined antiplatelet treatment with aspirin and clopidogrel demonstrated better results than aspirin alone, but was still worse than warfarin, with a $44 \%$ higher risk of vascular complications and a $30 \%$ higher risk of major bleeding. ${ }^{3}$ The goal of anticoagulation is to offer the patient a therapy that is better at preventing ischemic strokes at acceptable risk of bleeding; yet, the narrow therapeutic window of VKA results in a delicate balance between lack of efficacy and a significantly elevated bleeding risk. The variability in pharmacokinetics, leading to the necessity of frequent blood tests, and numerous food and drug interactions have a major impact on patient's daily life and furthermore lead to a relevant proportion of patients being either sub- or supratherapeutic. In a large study of almost 42,000 patients with NVAF (population ranging in age from 40 years to over 85 years), only $20 \%$ remained on warfarin for 6 years. ${ }^{4}$ NOACs brought the hope back for an effective and safe alternative to VKA due to better efficacy and far better compliance. A meta-analysis of 71,683 participants included in the Randomized Evaluation of Long-Term Anticoagulation Therapy (RE-LY), Rivaroxaban Once Daily Oral Direct Factor Xa Inhibition Compared with Vitamin K Antagonism for Prevention of Stroke and Embolism Trial in Atrial Fibrillation (ROCKET AF), Apixaban for Reduction in Stroke and Other Thromboembolic Events in Atrial Fibrillation (ARISTOTLE), and Effective Anticoagulation with Factor Xa Next Generation in Atrial Fibrillation -Thrombolysis in Myocardial Infarction 48 trial (ENGAGE AF-TIMI 48) trials showed that NOACs compared favorably with warfarin and significantly reduced the risk of stroke (relative risk [RR]: $0.81,95 \%$ confidence interval [CI]: 0.73-0.91; $P<0.0001$ ), and mortality (RR: $0.90,95 \% \mathrm{CI}$ : $0.85-0.95 ; P=0.0003)$. However, interestingly, low-dose regimens showed significantly more ischemic strokes (RR: $1.28,95 \%$ CI: $1.02-1.60 ; P=0.045) .{ }^{5} \mathrm{NOACs}$ had a favorable risk-benefit profile, even though with increased gastrointestinal bleeds. These findings could be, to some extent, anticipated as the patients at the highest risk of stroke and, therefore, with the greatest need for antithrombotic therapy are precisely those who experience more bleeding. ${ }^{6}$ Three of the clinical features that predict stroke (stroke history, hypertension, and advanced age) are also predictors of bleeds. It has been proved for warfarin, but seems to be true for NOACs as well. Anticoagulation-related bleeds may have devastating or lethal effect on patient outcome and cannot be fully eliminated. The presence of absolute or relative contraindications limits substantially the use of any anticoagulation drug in clinical practice due to perceived risk and fear to induce bleeding. ${ }^{7}$ Moreover, there are no data allowing safe restart of anticoagulation therapy in individuals with bleeding history. This clinical dilemma and a crucial question as well has not been addressed in Apixaban Versus Acetylsalicylic Acid to Prevent Stroke in Atrial Fibrillation Patients Who Have Failed or Are Unsuitable for Vitamin K Antagonist Treatment (AVERROES) trial, either. ${ }^{8}$ Although apixaban reduced the risk of stroke or systemic embolism by $55 \%$ without significantly increasing the risk of major bleeding, the highly limited representations of true bleeders (3\%) made it difficult to draw definite safety conclusions in patients with a history of bleeding.

\section{The rationale for the left atrial appendage occlusion}

The device-based therapies offer alternative approach to stroke and mortality prevention in NVAF population with the mission to "stop the stroke where it starts". The strategies to surgically eliminate or transcutaneously occlude left atrial appendage (LAA) are supported by the results of echocardiographic, surgical, and autoptic studies showing LAAs as the site for thrombus formation in $90 \%$ of patients with thrombosis owing to NVAF. ${ }^{910}$ In patients with NVAF, blood flow velocity in LAA frequently decreases, resulting in stasis, volume overload following cavity enlargement, and increased activity of the platelet adhesion molecules. ${ }^{11}$ Surgical experience in the exclusion of LAA has $>60$ years of history. The first percutaneous LAA occlusion in humans was performed in 2001..$^{12,13}$ The currently available LAA occluders, especially in experienced hands, fulfill high feasibility criteria. Studies report a success rate of $95 \%-96 \%$ if performed by experienced operators. ${ }^{14}$ The efficacy of the therapy has been proved by WATCHMAN Left Atrial Appendage Closure Device for Embolic PROTECTion in Patients with Atrial Fibrillation (PROTECT AF) study results, comparing LAA occlusion with warfarin. The criteria for the device noninferiority were met in short- and mid-term follow-up. The frequency of primary efficacy event rate occurrence (composite of stroke, cardiovascular death, and systemic embolism) was 3 and 4.3 per 100 patient-years in WATCHMAN and warfarin groups, respectively (rate ratio 0.71 with probability of noninferiority $>99.9 \%$ in the follow-up of 1,588 patient-years, mean of 2.3 years for a patient).$^{15}$ Moreover, the long-term follow-up (2,621 patient-years, mean of 4 years for a patient) 
demonstrated the superiority of the device with $40 \%$ RR reduction and $96 \%$ probability of device superiority. ${ }^{16}$ The reduction of the primary efficacy end point by WATCHMAN implantation was confirmed in intention-to-treat, postprocedure, per-protocol, and terminal therapy analyses. In addition, in an intention-to-treat analysis, patients were at reduced risk after LAA occlusion compared with warfarintreated patients for both all-cause mortality $(3.2 \%$ vs $4.8 \%$; hazards ratio [HR]: $0.66,95 \% \mathrm{CI}: 0.45-0.98 ; P=0.0379)$ and cardiovascular mortality $(1.0 \%$ vs $2.4 \%$; HR: $0.40,95 \% \mathrm{CI}$ : $0.23-0.82 ; P=0.0045) .{ }^{16}$ The safety issues of LAA occlusion were primarily of serious concern, along with the discouraging results of the early registries showing $7 \%-10 \%$ incidence of serious procedure-related complications. The comparison, however, of procedure/device-related events in the early and late phases of PROTECT AF and in continued access protocol (CAP) registry (performed by experienced operators) revealed decrease of complications: $10 \%$ versus $5.5 \%$ versus $3.7 \%$, respectively. The high risk of pericardial effusions in an early phase $(6.3 \%)$ dropped down to $3.7 \%$ in the late phase of the study and further down to $2.2 \%$ in the registry. Periprocedural strokes were eliminated $(1.1 \%$ vs $0.7 \%$ vs $0 \%))^{15,17}$ Proven procedural safety in experienced hands left the procedural learning curve as an issue that needed further evaluation. Consequently, the procedural learning curve evaluation was one of the aims of the Prospective Randomized Evaluation of the WATCHMAN LAA Closure Device in Patients With Atrial Fibrillation Versus Long Term Warfarin Therapy (PREVAIL) study, which required at least every fifth patient to be treated at centers not performing LAA occlusions before and also every fourth implantation performed by cardiologists inexperienced in LAA closure at each site. Actually, the PREVAIL study ended up with $39 \%$ of new recruiting sites and $39 \%$ of new operators. The implantation success rate and the rate of major adverse events remained unchanged, proving the efficacy of the newly implemented advanced training program. ${ }^{18}$ This training included online testing, mandatory 1-day professional training, along with observation of live cases, and experienced clinical specialists' support and proctoring before achieving final independence. The PREVAIL study reported low incidence of adverse events. The rate of pericardial effusion requiring pericardiocentesis was $1.5 \%$, tamponade requiring surgical repair $0.4 \%$, device/procedure-related stroke $0.4 \%$, and device embolization $0.8 \%$. Pathophysiological assumptions and clinical data derived from randomized studies paved the way toward rational implementation of the device-based therapy in the prevention of stroke and death in NVAF patient population.

\section{The inconsistency of data and concerns regarding LAA occlusion}

The data derived from the PROTECT AF trial sustained the view of the central role of LAA in the pathophysiology of stroke in patients with atrial fibrillation (AF). The results proved that eliminating the appendage may have profound impact on the long-term outcome. The equalized difference in the number of safety events between groups after 4 years of observation (3.6 vs 3.1 events per 100 patient-years; RR: $1.21,95 \%$ credible interval [CrI]: $0.78-1.94)$, equalized difference in the number of ischemic strokes (1.4 vs 1.1 events per 100 patient-years; RR: $1.26,95 \%$ CrI: $0.72-3.28)$, and higher number of hemorrhagic strokes in the controls $(0.2 \mathrm{vs}$ 11 events per 100 patient-years; RR: $0.15,95 \%$ CrI: 0.03 0.49 ), along with higher likelihood to die from hemorrhagic stroke while being on warfarin $(0.4 \%$ vs $2.9 \% ; P=0.0098)$ led to the conclusion that we have to wait 4 years to reach superiority of LAA closure in comparison to warfarin in terms of efficacy and equalize the risk. ${ }^{16}$ The long-lasting interaction between the manufacturer and the US Food and Drug Administration (FDA) Circulatory System Devices Panel, however, sheds light on the lack of data consistency. ${ }^{19}$ The FDA panel questioned the high hemorrhagic stroke rate in PROTECT AF that drove the benefit of the WATCHMAN device over the control group. The hemorrhagic stroke rate was substantially higher than observed in contemporary anticoagulation trials ( $0.4 \mathrm{vs} 1.1)$ and raised questions regarding the robustness of this potential benefit. The FDA also questioned the reduction in bleeding events in the WATCHMAN group because when accounting for bleeding associated with implantation procedure, there was no overall difference in the bleeding rates. Moreover, a disproportionately increased rate of subject withdrawals in the control group in PROTECT AF reduced the robustness of these findings as well. The rate of withdrawal of consent was nearly fivefold greater in the control group (18\%) versus the device group (3.7\%). The disparity in withdrawal rates between treatment groups could lead to bias against the control group and favor the device group for the long-term event rate. The results of PREVAIL study intensified concerns. Based on the prespecified hypothesis during 18-month follow-up, the device did not appear to be noninferior to anticoagulation therapy according to the first primary study end point $(0.064$ vs 0.063 ; RR: $1.07,95 \%$ CrI: $0.57-1.89)$. The range of $95 \%$ $\mathrm{CrI}$ exceeded the level of noninferiority margin prespecified in the protocol. ${ }^{18}$ The update with the data from the longterm follow-up of the PREVAIL study resulted with a total of 13 ischemic strokes in the WATCHMAN group and one 
in the control group. The new ischemic strokes occurred in subjects whose device implantations were performed over 1 year before and substantially changed the assessment of the benefit-risk profile of the WATCHMAN device, raising concerns regarding the effectiveness of the device and favoring the control group. ${ }^{19}$ Overall, there was a further divergence in the rate of ischemic stroke and systemic embolism, whereas the hemorrhagic strokes were infrequent (two events in each treatment group). The rate of cardiovascular or unexplained death, yet, numerically favored the device group, but none of the deaths was causally linked to the WATCHMAN device, implantation procedure, or anticoagulant therapy. After adoption of long-term PREVAIL follow-up data, the device no longer met the second primary end point and still has not met the first primary end point of the study. ${ }^{19}$ PROTECT AF and PREVAIL appeared to be diverging. The late increase in WATCHMAN-associated thromboembolic events and loss of its noninferiority compared to warfarin for non-procedure-related ischemic events (event rate 0.029 vs 0.013 , respectively) raised concern if these late ischemic strokes may be related to late thrombus formation on the WATCHMAN device in the absence of anticoagulation. It is, however, possible that many strokes were unrelated to LAA thrombi. AF may also be associated with hypertension, carotid artery atherosclerosis, or systemic inflammatory and hypercoagulable state unrelated to the presence or absence of LAA thrombi and independently leading to stroke. In two studies, for instance, evaluating the correlation between AF burden and thromboembolism using implantable pacemakers and defibrillators with atrial leads to accurately and continuously record AF burden, only $10 \%-30 \%$ of patients who developed thromboembolism had AF detected within 1 month of their event.. ${ }^{20,21}$ At this point, many questions surrounding the safety and longterm efficacy of WATCHMAN remain unanswered. The concerns about WATCHMAN require even greater caution in assessing the efficacy of different appendage occluding devices. It is crucial to emphasize that WATCHMAN is the best-studied device, evaluated in the randomized studies against controls, in contrast with other occluders that were evaluated solely in registries against presumed risk derived from risk-score calculations. The access to only limited data does not allow for confidence and leaves us with even less certainty about their efficacy and safety. Having said that, the task of discussing proper patient selection for LAA occlusion is challenging indeed.

It is crucial to mention at this point that despite the earlier discussed concerns, there was a mixed response in the opinion of experts constituting the FDA panel. The panel was mostly in favor of using the totality of the data, instead of just looking that PREVAIL did not meet the first primary end point. There was a consensus in the panel that the device has a role in a specific group of patients, suggesting that the device would be used as a second-line therapy for appropriate patients, and the panel members finally voted 12 to 0 that the WATCHMAN LAA closure device is safe; six to seven that it is not effective; and six to five, with one abstention, that its benefits outweigh its risks. The panel recommendations were not binding to the FDA and in March 2015, WATCHMAN was FDA approved for use in the US. The device is indicated to reduce the risk of thromboembolism from LAA in patients with NVAF who are at an increased risk of stroke and systemic embolism based on CHADS2 or CHADS2-VASc scores, are deemed by their physicians to be suitable for warfarin, and have appropriate rationale to seek a nonpharmacologic alternative to warfarin, taking into account the safety and effectiveness of the device compared to warfarin.

\section{The key points in patient selection for LAA occlusion}

According to the WATCHMAN 2005 first CE marking, the device was registered to prevent thrombus embolization from LAA and reduce the risk of life-threatening bleeding events in patients with NVAF who were eligible for anticoagulation therapy. This registration reflected precisely the population of patients recruited into both milestone trials of LAA closure. Similarly, the FDA 2015 device registration referred to patients at increased risk of stroke and bleeds, who were still eligible for anticoagulation. From this perspective, the LAA occlusion might be addressed to patients with NVAF at bleeding risk who feel uncomfortable with the perspective of lifelong systemic anticoagulation therapy or patients with stroke recurrence under anticoagulation therapy, but not for individuals with persistent noncompliance or nontolerability or those simply refusing their intake. Theoretically, the ideal candidate for LAA closure, however, would be a patient with absolute contraindications for anticoagulation, deemed with a history of major bleeding (especially gastrointestinal), or a patient with the history of hemorrhagic stroke. Although the 2012 WATCHMAN CE marking expanded the device registration for patients with contraindication to anticoagulation and the European Society of Cardiology (ESC) supported this indication by recommending LAA occlusion in patients with a high stroke risk and with contraindications for long-term oral anticoagulation (class IIb/B), it must be noted that this recommendation is not derived from any randomized study. ${ }^{22}$ In fact, no direct comparison between LAA occlusion and control group without anticoagulation 
has been done in a randomized model so far. Even though it is theoretically and intuitively clinically attractive, the safety and efficacy of WATCHMAN in patients who are poor candidates for even short-term anticoagulant or antiplatelet therapy remain currently unproven. Certainly, the ASA Plavix Feasibility Study With Watchman Left Atrial Appendage Closure Technology (ASAP) registry has no power to fill this gap. ${ }^{23}$ This is unfortunate, as patients who are unable or unwilling to take warfarin are the patient populations that would likely derive the greatest benefit from WATCHMAN. On the top of divergences of the previously discussed recommendations and in contrary to FDA opinion, the British National Health Service Commissioning Board ruled that the cost-effectiveness and clinical effectiveness of the device are not established enough and, therefore, the device is not funded in the UK, based on an emerging report on the costeffectiveness of LAA occlusion. ${ }^{24}$

\section{The current everyday practice in patient selection for LAA closure}

In the majority of labs in Europe, the patient selection practice is based on device's CE marking in accordance with the expert consensus statement published in 2014 by the European Heart Rhythm Association and the European Association of Percutaneous Cardiovascular Interventions. ${ }^{25}$ According to this document, contrary to FDA registration and ESC 2012 recommendations, LAA closure might be considered in a broad spectrum of NVAF patients; however, the expert statement underlines the crucial role of patient participation in decision making after receiving comprehensive data on feasibility, efficacy, and safety of implantation in contrast to long-term anticoagulation therapy and no treatment.

\section{LAA occluder implantation as an alternative to oral anticoagulation when oral anticoagulation is possible}

LAA occlusion should be mentioned to the patient; however, it must be stressed that anticoagulation currently remains the standard of therapy with much more supporting evidence than LAA occluders.

\section{LAA occluder implantation as a replacement of anticoagulation therapy when anticoagulation is not possible due to contraindications}

The most accepted clinical indications for LAA occlusion are life-threatening bleeds and significant bleeds, the source of which could not be eliminated. This statement is based on expert consensus derived from several observational studies and registries, as no randomized data targeting this specific group of patients are available. While discussing the treatment strategy with the patient, it is mandatory to mention that dual antiplatelet therapy generates a major bleeding risk comparable to that of warfarin. However, exposure following LAA occlusion is only for a short time, reducing the cumulative risk. In patients who cannot receive any antiplatelet agent, transepicardial LAA ligation can be considered.

\section{LAA occluder implantation as a replacement of anticoagulation therapy in case of increased bleeding risk under systemic anticoagulation}

The decision to implant LAA occluder results from an individual risk-benefit evaluation. LAA occlusion has to be presented as an alternative to anticoagulation. NOACs or anticoagulation-free therapy has to be considered before final decision is taken. Patients with an increased HASBLED score or who are at higher bleeding risk not well characterized by the HAS-BLED score but resulting from other clinical circumstances or patients who require double antiplatelet therapy and patients with end-stage renal failure constitute this subgroup of potential candidates. It should be taken into account that for at least 1-6 months, either anticoagulants or double antiplatelet therapy is warranted after LAA occlusion.

\section{LAA occluder implantation as a complement to anticoagulation}

The combination of LAA occlusion and sustained long-term anticoagulation therapy is occasionally performed in patients with embolic events despite achieving adequate level of anticoagulation. The LAA occlusion could be debated as an alternative treatment, especially when AF-related embolism occurs while taking VKA with documented elevated International Normalized Ratios (INRs) or embolic episode occurred on NOACs or switching to NOACs is not possible due to contraindication; however, there are no data available which would support this approach.

\section{Conclusion}

The divergence of results between the two main randomized clinical trials evaluating the safety and efficacy of LAA closure device limited substantially the credibility of data promoting vast utilization of this technology in a broad spectrum of patients requiring embolic prevention therapy in the course of NVAF. The lack of certainty according to safety issues of LAA occluder implantation had been replaced by the lack 
of certainty according to long-term efficacy of local devicebased therapy. On the other hand, the diverse interpretation of the totality of published data including randomized trials, registries, cost-effectiveness analysis, and personal experts' experiences means that the indications for this treatment modality are not uniform and vary substantially between continents and from country to country in Europe. At this stage, the LAA closure technology, although registered by FDA and labeled by CE mark and ESC recommendations, is, in fact, at the crossroads and requires further properly designed prospective, randomized evaluation involving different occluders in broader, specifically addressed spectrum of clinical presentations.

\section{Disclosure}

Lectures fee sponsored by Boston Scientific. The author reports no other conflicts of interest in this work.

\section{References}

1. Miyasaka Y, Barnes ME, Gersh BJ, et al. Secular trends in incidence of atrial fibrillation in Olmsted County, Minnesota, 1980 to 2000, and implications on the projections for future prevalence. Circulation. 2006;114(2):119-125.

2. Hart RG, Pearce LA, Aguilar MI. Meta-analysis: antithrombotic therapy to prevent stroke in patients who have nonvalvular atrial fibrillation. Ann Intern Med. 2007;146(12):857-867.

3. Connolly S, Pogue J, Hart R, et al; the ACTIVE Writing Group of the ACTIVE Investigators. Clopidogrel plus aspirin versus oral anticoagulation for atrial fibrillation in the Atrial fibrillation Clopidogrel Trial with Irbesartan for prevention of Vascular Events (ACTIVE W): a randomized controlled trial. Lancet. 2006;367(9526):1903-1912.

4. Gallagher AM, Rietbrock S, Plumb J, van Staa TP. Initiation and persistence of warfarin or aspirin in patients with chronic atrial fibrillation in general practice: do the appropriate patients receive stroke prophylaxis? J Thromb Haemost. 2008;6(9):1500-1506.

5. Ruff CT, Giugliano RP, Braunwald E, et al. Comparison of the efficacy and safety of new oral anticoagulants with warfarin in patients with atrial fibrillation: a meta-analysis of randomised trials. Lancet. 2014;383(9921):955-962.

6. Hylek EM, Evans-Molina C, Shea C, Henault LE, Regan S. Major hemorrhage and tolerability of warfarin in the first year of therapy among elderly patients with atrial fibrillation. Circulation. 2007;115(21): 2689-2696.

7. Nieuwlaat R, Capucci A, Camm AJ, et al. Atrial fibrillation management: a prospective survey in ESC Member Countries: The Euro Heart Survey on Atrial Fibrillation. Eur Heart J. 2005;26(22):2422-2434.

8. Connolly SJ, Eikelboom J, Joyner C, et al. Apixaban in patients with atrial fibrillation. (AVERROES trial). N Engl J Med. 2011;364(9):806-817.

9. Manning WJ, Weintraub RM, Waksmonski CA, et al. Accuracy of transesophageal echocardiography for identifying left atrial thrombi: a prospective, intraoperative study. Ann Intern Med. 1995;123(11):817-822.

10. Blackshear JL, Odell JA. Appendage obliteration to reduce stroke in cardiac surgical patients with atrial fibrillation. Ann Thorac Surg. 1996;61(2):755-759.
11. Fukuchi M, Watanabe J, Kumagai K, et al. Increased von Willebrand factor in the endocardium as a local predisposing factor for thrombogenesis in overloaded human atrial appendage. J Am Coll Cardiol. 2001;37(5):1436-1442.

12. Madden JL. Resection of the left auricular appendix: a prophylaxis for recurrent arterial emboli. J Am Med Assoc. 1949;140(9):769-772.

13. Sievert H, Lesh MD, Trepels T, et al. Percutaneous left atrial appendage transcatheter occlusion to prevent stroke in high-risk patients with atrial fibrillation: early clinical experience. Circulation. 2002;105(16):1887-1889.

14. Reddy V.Y, Holmes D, Doshi SK, Neuzil P, Kar S. Safety of percutaneous left atrial appendage closure. Results from the Watchman Left Atrial Appendage System for Embolic Protection in Patients with AF (PROTECT AF) Clinical Trial and the Continued Access Registry. Circulation. 2011;123(4):417-424.

15. Reddy VY, Doshi SK, Sievert H, et al. Percutaneous left atrial appendage closure for stroke prophylaxis in patients with atrial fibrillation: 2.3year follow-up of the PROTECT AF (Watchman Left Atrial Appendage System for Embolic Protection in Patients with Atrial Fibrillation) Trial. Circulation. 2013;127(6):720-729.

16. Reddy VY, Sievert H, Halperin J, et al. Percutaneous left atrial appendage closure vs warfarin for atrial fibrillation: a randomized clinical trial. PROTECT AF Steering Committee and Investigators. JAMA. 2014;312(19):1988-1998.

17. Gangireddy SR, Halperin JL, Fuster V, Reddy VY. Percutaneous left atrial appendage closure for stroke prevention in patients with atrial fibrillation: an assessment of net clinical benefit. Eur Heart J. 2012;33(21):2700-2708.

18. Holmes DR, Kar S, Price MJ, et al. Prospective randomized evaluation for the WATCHMAN left atrial appendage closure device in patients with atrial fibrillation versus long-term warfarin therapy: the PREVAIL trial. J Am Coll Cardiol. 2014;64(1):1-12.

19. Waksman R, Pendyala LK. Overview of the Food and Drug Administration circulatory system devices panel meetings on WATCHMAN left atrial appendage closure therapy. Am J Cardiol. 2015;115(3):378-384.

20. Daoud EG, Glotzer TV, Wyse DG, et al. Temporal relationship of atrial tachyarrhythmias, cerebrovascular events, and systemic emboli based on stored device data: a subgroup analysis of TRENDS. Heart Rhythm. 2011;8(9):1416-1423.

21. Brambatti M, Connolly SJ, Gold MR, et al. Temporal relationship between subclinical atrial fibrillation and embolic events. Circulation. 2014;129(21):2094-2099.

22. Camm AJ, Lip GY, De Caterina R, et al. 2012 focused update of the ESC guidelines for the management of atrial fibrillation. Eur Heart J. 2012;33(21):2719-2747.

23. Reddy VY, Mobius-Winkler S, Miller MA, et al. Left atrial appendage closure with the WATCHMAN device in patients with a contraindication for oral anticoagulation: the ASAP study (ASA Plavix Feasibility Study with WATCHMAN Left Atrial Appendage Closure Technology). J Am Coll Cardiol. 2013;61(25):2551-2556.

24. Clinical Commissioning Policy Statement: Left Atrial Appendage (LAA) Occlusion. Commissioning Board: National Health Service; 2013.

25. Meier B, Blaauw Y, Khattab AA, et al. EHRA/EAPCI expert consensus statement on catheter-based left atrial appendage occlusion. Europace. 2014;16(10):1397-1416. 
Research Reports in Clinical Cardiology

Dovepress

\section{Publish your work in this journal}

Research Reports in Clinical Cardiology is an international, peerreviewed, open access journal publishing original research, reports, editorials, reviews and commentaries on all areas of cardiology in the clinic and laboratory. The manuscript management system is completely online and includes a very quick and fair peer-review system.

Submit your manuscript here: http://www.dovepress.com/research-reports-in-clinical-cardiology-journal
Visit http://www.dovepress.com/testimonials.php to read real quotes from published authors. 